\title{
Autosomal recessive polycystic kidney disease and congenital hepatic fibrosis (ARPKD/CHF)
}

\author{
Baris Turkbey, \\ Molecular Imaging Program, National Cancer Institute, National Institutes of Health, Bethesda, \\ MD, USA \\ Iclal Ocak, \\ Molecular Imaging Program, National Cancer Institute, National Institutes of Health, Bethesda, \\ MD, USA
}

\section{Kailash Daryanani,}

Clinical Center, Department of Radiology, National Institutes of Health, Bethesda, MD, USA

\section{Esperanza Font-Montgomery,}

National Human Genome Research Institute, Medical Genetics Branch, National Institutes of Health, 10 Center Drive, Building 10, Bethesda, MD 20892, USA

\section{Linda Lukose,}

National Human Genome Research Institute, Medical Genetics Branch, National Institutes of Health, 10 Center Drive, Building 10, Bethesda, MD 20892, USA

\section{Joy Bryant,}

National Human Genome Research Institute, Medical Genetics Branch, National Institutes of Health, 10 Center Drive, Building 10, Bethesda, MD 20892, USA

\section{Maya Tuchman,}

National Human Genome Research Institute, Medical Genetics Branch, National Institutes of Health, 10 Center Drive, Building 10, Bethesda, MD 20892, USA

\section{Parvathi Mohan, \\ Department of Pediatric Gastroenterology, George Washington University, Washington, DC, USA \\ Theo Heller, \\ National Institute of Diabetes and Digestive and Kidney Diseases, National Institutes of Health, Bethesda, MD, USA}

\section{William A. Gahl,}

National Human Genome Research Institute, Medical Genetics Branch, National Institutes of Health, 10 Center Drive, Building 10, Bethesda, MD 20892, USA

Peter L. Choyke, and Molecular Imaging Program, National Cancer Institute, National Institutes of Health, Bethesda, MD, USA

\section{Meral Gunay-Aygun}

National Human Genome Research Institute, Medical Genetics Branch, National Institutes of Health, 10 Center Drive, Building 10, Bethesda, MD 20892, USA mgaygun@mail.nih.gov

\footnotetext{
(C) Springer-Verlag 2008

Correspondence to: Meral Gunay-Aygun.

Present address: I. Ocak Department of Radiology, University of Pittsburgh Medical Center, Pittsburgh, PA, USA
} 
Intramural Program, Office of Rare Diseases, Office of the Directors, National Institutes of Health, Bethesda, MD, USA

\section{Abstract}

ARPKD/CHF is an inherited disease characterized by non-obstructive fusiform dilatation of the renal collecting ducts leading to enlarged spongiform kidneys and ductal plate malformation of the liver resulting in congenital hepatic fibrosis. ARPKD/CHF has a broad spectrum of clinical presentations involving the kidney and liver. Imaging plays an important role in the diagnosis and follow-up of ARPKD/CHF. Combined use of conventional and high-resolution US with MR cholangiography in ARPKD/CHF patients allows detailed definition of the extent of kidney and hepatobiliary manifestations without requiring ionizing radiation and contrast agents.

\section{Keywords}

Autosomal recessive polycystic kidney disease; Congenital hepatic fibrosis; US; MR cholangiography; Children

\section{Introduction}

Autosomal recessive polycystic kidney disease/congenital hepatic fibrosis (ARPKD/CHF) is an inherited hepatorenal fibrocystic disease with an estimated frequency of 1 in 20,000 live births [1]. It is characterized by non-obstructive fusiform dilatations of the renal collecting ducts resulting in enlarged spongiform kidneys and ductal plate malformation of the liver leading to CHF/Caroli syndrome (Fig. 1) [2]. Most patients with ARPKD/CHF present perinatally with oligohydramnios caused by decreased fetal urine output and related hypoplastic lungs, but others present later in life when the clinical symptomatology is dominated by either renal failure or hepatic dysfunction, or both. Both kidney and liver disease are progressive. For instance, almost all patients with ARPKD have some degree of $\mathrm{CHF}$ at birth, but in some individuals this leads to portal hypertension (PH) which is characterized by splenomegaly, hypersplenism with low platelet counts, gastroesophageal variceal bleeding, or cholangitis of variable severity as they get older. Patients with milder kidney disease come to medical attention later in childhood or in adulthood with manifestations of either $\mathrm{PH}$ or chronic renal insufficiency requiring renal replacement therapy. Biliary cystic disease, often referred to as Caroli syndrome, frequently accompanies CHF and predisposes children to cholangitis caused by biliary stasis. In addition, CHF patients are at increased risk of benign and malignant liver tumors, especially cholangiocarcinoma. Although molecular genetic testing has recently become available to confirm diagnosis of ARPKD/CHF, the diagnosis continues to depend largely on imaging because of the large and complex nature of the PKHDl gene.

\section{Genetic basis and pathophysiology}

ARPKD/CHF is caused by mutations in the PKHDI gene located on chromosome $6 \mathrm{p} 12$. $P K H D 1$ is a relatively large gene containing 66 coding exons, which demonstrates a complex splicing pattern. The gene codes for the protein fibrocystin, a hepatocyte growth factor receptor-like protein that functions on the primary cilia of renal and biliary epithelial cells (Fig. 2) [3]. The most severe form of ARPKD/CHF involves two protein-truncating mutations, while milder forms of the disease typically have one or more missense mutations [4]. Dysfunction of fibrocystin leads to abnormal ciliary signaling, which is normally required for regulation of proliferation and differentiation of renal and biliary epithelial cells. 


\section{Clinical manifestations}

Clinical manifestations depend on the relative severity of kidney and liver disease. In the most severe perinatal form of ARPKD/CHF, the kidneys are markedly enlarged because of the cumulative effect of dilatation of all of the medullary and cortical collecting ducts. In severe prenatal cases, involvement of the kidneys leads to oligohydramnios and pulmonary hypoplasia associated with Potter facies. Although most infants with ARPKD/CHF have an elevated serum creatinine, oliguria and hyponatremia during the first days of life, impaired renal function is often temporary and perinatal survival depends largely on the degree of pulmonary hypoplasia [5]. Respiratory distress and pneumothorax often complicate pulmonary hypoplasia and worsen the clinical picture. The prognosis of ARPKD/CHF in patients presenting soon after birth is now better than described in the earlier literature. Although most infants presenting in the perinatal period ultimately require renal transplantation, the age at transplantation is very variable and can be occasionally delayed until adulthood. Severe systemic hypertension, often diagnosed at birth, is present in approximately $80 \%$ of patients. Individuals who survive their neonatal respiratory complications and those who present with liver-predominant symptoms later in childhood develop variable degrees of $\mathrm{PH}$ and chronic renal failure as they age. The rates of progression of hepatic and renal disease can vary, even among patients carrying the same PKHDl mutation, and are independent of each other. Thus, current mutation analysis is not necessarily predictive of outcome.

\section{Imaging protocol}

Our imaging protocol for ARPKD/CHF includes the integrated use of US and MRI both at baseline and during follow-up visits, which occur every 1-2 years or sooner if medically necessary. This avoids both contrast medium administration and ionizing radiation in these young patients. US scans are performed with conventional 4-MHz and high-resolution 6- to 9-MHz probes following 3-6 $\mathrm{h}$ of fasting, depending on the age of the patient. Our MR images were obtained on a 1.5-T scanner (GE CV/I, General Electric Medical Systems, Waukesha, WI, and Philips Achieva, Philips Medical Systems, Best, The Netherlands) with the cardiac coil. The MR imaging protocol includes axial and coronal T1-weighted (T1-W) and T2-weighted (T2-W) images and MR cholangiography (with thin and thick slab imaging parallel to the common bile duct). MR cholangiography images are postprocessed using a maximum-intensity projection algorithm to obtain views of the entire biliary tree from different angles.

\section{Imaging findings}

Kidneys-Sonography is the diagnostic method of choice for assessing the kidneys in young children. In newborns and infants, the kidneys can be markedly enlarged with a preserved reniform contour (Fig. 3). On US at conventional insonating frequencies (3-5 $\mathrm{MHz}$ ), the enlarged kidneys are diffusely hyperechogenic, with loss of the normal corticomedullary distinction, and the cortex can appear as a thin hypoechoic rim at the periphery (Fig. 4). The diffuse medullary and cortical hyperechogenicity is caused by dilated collecting ducts, most of which maintain their continuity with the nephrons and have urine flow in the lumen, and can only be resolved on higher frequency scans (7-9 MHz). As patients age, some of these ducts become enclosed cysts that enlarge, resulting in scattered medullary and cortical macrocysts superimposed on the background of diffuse hyperechogenicity. However, unlike in autosomal dominant polycystic kidney disease (ADPKD), these cysts are rarely complicated by hemorrhage or infection. Another major difference between the kidneys in ARPKD/CHF and ADPKD is the growth of the kidneys over time. In typical ARPKD/CHF, the kidneys are markedly enlarged before birth and continue to grow in the first 2-3 years of life; thereafter, kidney growth plateaus or even 
decreases. In contrast, kidneys associated with ADPKD demonstrate progressive growth throughout life as the size and number of macrocysts increase.

During our assessment of ARPKD/CHF patients with high-resolution (7-9 MHz) transducers, we characterized the patients into groups according to amount of renal involvement. Some patients had only partial medullary involvement, while others demonstrated complete medullary involvement with $<50 \%$ cortical involvement or complete medullary involvement with $>50 \%$ cortical involvement. This pattern-basedapproachallows amore accurate classification of the extent of renal manifestations and a more uniform description of the complex changes occurring in the kidneys (Figs. 5, 6, 7 and 8). In almost all of our ARPKD/CHF patients with partial medullary involvement, US examinations of the kidney performed with a standard 4-MHz transducer were normal; only high-resolution US with a 6- to $9-\mathrm{MHz}$ probe enabled detection of subtle ductal dilatations confined to the medulla [6]. In our cohort, all patients with partial medullary involvement had a normal creatinine clearance, whereas those with more severely affected kidneys on sonography had an abnormal creatinine clearance. Thus, the sonographic appearance, particularly the preservation of the normal renal cortex, does roughly correlate with the renal function.

An additional common sonographic finding in ARPKD/CHF kidneys is the presence of numerous non-shadowing echogenic foci. These small foci do not display acoustic shadowing and thus are not renal stones. Although the origins of the foci are uncertain, Lucaya et al. [7] suggested that they may represent urine stagnation within dilated tubules including calcium precipitation secondary to diminished citrate excretion (Fig. 9). The majority of our patients had echogenic foci, but the 24-hour urinary excretion of calcium was normal in all of them. An alternative explanation is that the multiple sharp interfaces between dilated collecting ducts causes artefactual echogenic foci. However, the precise nature of the echogenic foci remains to be determined.

On CT, the kidneys are low in attenuation because of their high water content. Following intravenous contrast material administration, the kidneys have a striking striated appearance as the excreted contrast material slowly fills the dilated collecting ducts (Fig. 10).

MRI can demonstrate enlarged kidneys that are darker than normal on T1-W images and brighter than normal on T2-W images owing to the large amount of water in the dilated ducts within the kidneys (Fig. 11). The degree of cortical involvement and cystic complications is apparent on a combination of T1-W and T2-W images. MR imaging has been used in the prenatal diagnosis of ARPKD/CHF [8]. Fetal ARPKD/CHF kidneys appear enlarged and hyperintense on T2-W images.

Hepatobiliary system-Although all ARPKD/CHF patients including newborns have $\mathrm{CHF}$ at least at a microscopic level, imaging findings of CHF are often not detectable until later, sometimes first manifesting in adulthood. Findings associated with CHF on US include increased liver echogenicity, splenomegaly and intra- and extrahepatic biliary cysts/ dilatations. Liver echogenicity is diffusely increased and the parenchymal echotexture is coarsened, especially on high-resolution scans (Fig. 12). Periportal thickening caused by fibrosis can be readily demonstrated on US (Fig. 13). In addition to CHF, macroscopic cystic or fusiform dilatations of the intra- and extrahepatic bile ducts can be identified sonographically but only on high-resolution scans.

Real-time evaluation with US allows assessment of the intra- and extrahepatic biliary system. Macrocystic dilatations of the small peripheral or larger central intrahepatic biliary ducts (Caroli syndrome) were detected in majority of our patients. Dilated biliary ducts in Caroli disease are usually seen as macrocysts within the liver parenchyma; it is often 
challenging to confirm their continuity with the biliary tree using US (Fig. 14). The simultaneous presence of CHF with Caroli disease is termed Caroli syndrome (Fig. 15). Dilated extrahepatic bile ducts and an elongated hydropic gallbladder is another underrecognized feature of ARPKD/CHF [9]. We detected a dilated common bile duct and an enlarged gallbladder in $55 \%$ of patients in our own cohort.

Color Doppler US enables hemodynamic evaluation of the portal venous system, specifically showing the direction of portal blood flow, and varicose venous collaterals including recanalized umbilical veins (Fig. 16). Color Doppler imaging also allows detection of multiple vessels around the porta hepatis, resembling cavernous transformation despite a patent portal vein lumen; because of their over-crowded nature it is often impossible to determine the flow dynamics within these structures (Fig. 17). Interestingly, these cavernous transformation-like periportal vessels probably represent a developmental duplication of hepatic vascular branching that is part of CHF and is not an acquired pathology since it is usually present before the onset of $\mathrm{PH}$ and is not associated with occlusion of the portal vein [10].

The liver often has an abnormal shape with a disproportionately large left lobe extending down the midline toward the umbilicus and between the diaphragm and the spleen on the left. This is easily visible on CT or MRI and is consistent with the clinical observation that most CHF livers are easily palpable from the xiphoid down to the lower end of the costal margin, but they are generally not palpable at the right costal margin.

Although CT does not provide a complete parenchymal evaluation of the liver in CHF, it can demonstrate the abnormal size and shape of the liver. Periportal thickening is seen as decreased periportal attenuation; varices and splenomegaly can be visualized on CT (Fig. 18). In Caroli disease, the central dot sign, which represents the portal vein branch at the center of a dilated intrahepatic bile duct, can be readily demonstrated on CT and MRI images [11]. The major limitation to using CT in imaging of CHF patients is that renal insufficiency might preclude the administration of contrast medium. Careful blood and urine laboratory examinations are needed before CT imaging. The information provided by MRI is usually the same as or more than that provided by $\mathrm{CT}$ without the ionizing radiation and thus is usually preferred.

MR has recently been employed to evaluate the liver and biliary tree in the setting of ARPKD/CHF. Besides conventional MR imaging sequences (T1-W and T2-W), a heavily T2-W turbo spin-echo MR cholangiographic sequence allows noninvasive detection of the entire biliary system [12]. Intrahepatic biliary ductal dilatation can be demonstrated on axial, coronal and reformatted MR cholangiography images (Fig. 19). A distinctive feature of CHF MR cholangiograms is that the ducts do not taper to the periphery of the liver as they do normally. Instead, they remain dilated throughout the parenchyma, often forming cisterns and small cysts at the periphery of the liver (Fig. 20). MR cholangiography is also useful for detecting biliary system complications including stones and cholangitis. Fibrosis of the liver parenchyma appears as patchy, heterogeneous signal changes on T2-W images. In addition, periportal fibrosis demonstrates high signal intensity along the course of the portal vein branches. Findings of PH such as collaterals and hepatosplenomegaly can also be seen.

\section{Conclusion}

ARPKD/CHF demonstrates a spectrum of clinical and imaging presentations with variably severe renal and hepatic findings. US is easier to perform in unsedated children and yields the real-time data needed to characterize kidney and liver pathology. High-resolution US is particularly useful in assessing the extent of renal involvement and demonstrating the 
morphology of the hepatic parenchyma and biliary system. The major advantage of MR imaging is that MR cholangiography provides an overall depiction of the biliary tree, which is often dilated to the hepatic periphery. Combined use of high-resolution US and MR cholangiography allows detailed definition of the extent of kidney and hepatobiliary involvement without requiring contrast medium or ionizing radiation.

\section{Acknowledgments}

We thank the ARPKD/CHF Alliance and all patients and their families who generously participated in this investigation. Supported by the intramural research programs of the National Human Genome Research Institute, National Cancer Institute, National Institute of Diabetes and Digestive and Kidney Diseases, and the National Institutes of Health Clinical Center.

\section{References}

1. Zerres K, Mücher G, Becker J, et al. Prenatal diagnosis of autosomal recessive polycystic kidney disease (ARPKD): molecular genetics, clinical experience, and fetal morphology. Am J Med Genet 1998;76:137-144. [PubMed: 9511976]

2. Avner ED, Sweeney WE Jr. Renal cystic disease: new insights for the clinician. Pediatr Clin North Am 2006;53:889-909. [PubMed: 17027616]

3. Ward CJ, Hogan MC, Rossetti S, et al. The gene mutated in autosomal recessive polycystic kidney disease encodes a large, receptor-like protein. Nat Genet 2002;30:259-269. [PubMed: 11919560]

4. Bergmann C, Senderek J, Sedlacek B, et al. Spectrum of mutations in the gene for autosomal recessive polycystic kidney disease (ARPKD/PKHD1). J Am Soc Nephrol 2003;14:76-89. [PubMed: 12506140]

5. Sumfest JM, Burns MW, Mitchell ME. Aggressive surgical and medical management of autosomal recessive polycystic kidney disease. Urology 1993;42:309-312. [PubMed: 8379031]

6. Traubici J, Daneman A. High-resolution renal sonography in children with autosomal recessive polycystic kidney disease. AJR 2005;184:1630-1633. [PubMed: 15855129]

7. Lucaya J, Enriquez G, Nieto J, et al. Renal calcifications in patients with autosomal recessive polycystic kidney disease: prevalence and cause. AJR 1993;160:359-362. [PubMed: 8424350]

8. Liu YP, Cheng SJ, Shih SL, et al. Autosomal recessive polycystic kidney disease: appearance on fetal MRI. Pediatr Radiol 2006;36:169. [PubMed: 16283284]

9. Goilav B, Norton KI, Satlin LM, et al. Predominant extrahepatic biliary disease in autosomal recessive polycystic kidney disease: a new association. Pediatr Transplant 2006;10:294-298. [PubMed: 16677351]

10. Desmet VJ. Congenital diseases of intrahepatic bile ducts: variations on the theme "ductal plate malformation". Hepatology 1992;16:1069-1083. [PubMed: 1398487]

11. Zeitoun D, Brancatelli G, Colombat M, et al. Congenital hepatic fibrosis: CT findings in 18 adults. Radiology 2004;231:109-116. [PubMed: 14990822]

12. Pavone P, Laghi A, Catalano C, et al. Caroli's disease: evaluation with MR cholangiopancreatography (MRCP). Abdom Imaging 1996;21:117-119. [PubMed: 8661756] 
a

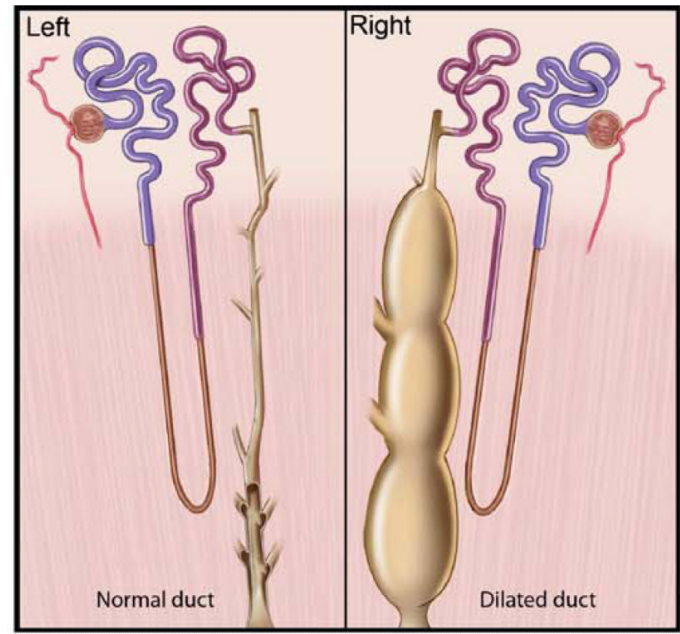

b

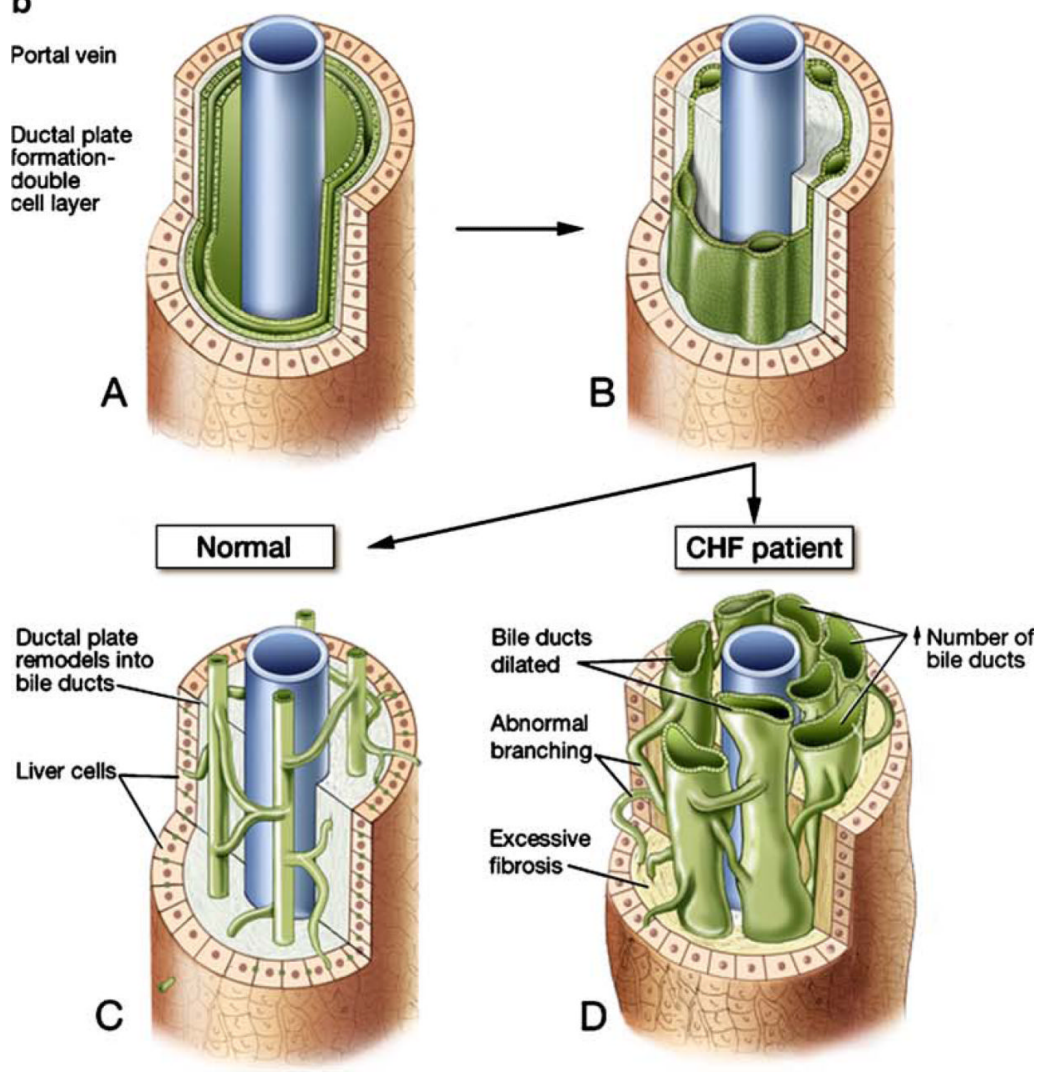

Fig. 1.

Normal and abnormal renal collecting ducts and biliary ductal plate. a Drawings demonstrate the normal structure of a renal collecting duct (left) and an abnormally dilated collecting duct of ARPKD (right). b The flow chart illustrates the normal development of the ductal plate from the double cell layer $(A, B)$ to the bile ducts around the portal vein $(C)$ and abnormal ductal plate remodeling $(D)$, which results in dilated bile ducts with abnormal branching and fibrosis in $\mathrm{CHF}$ 


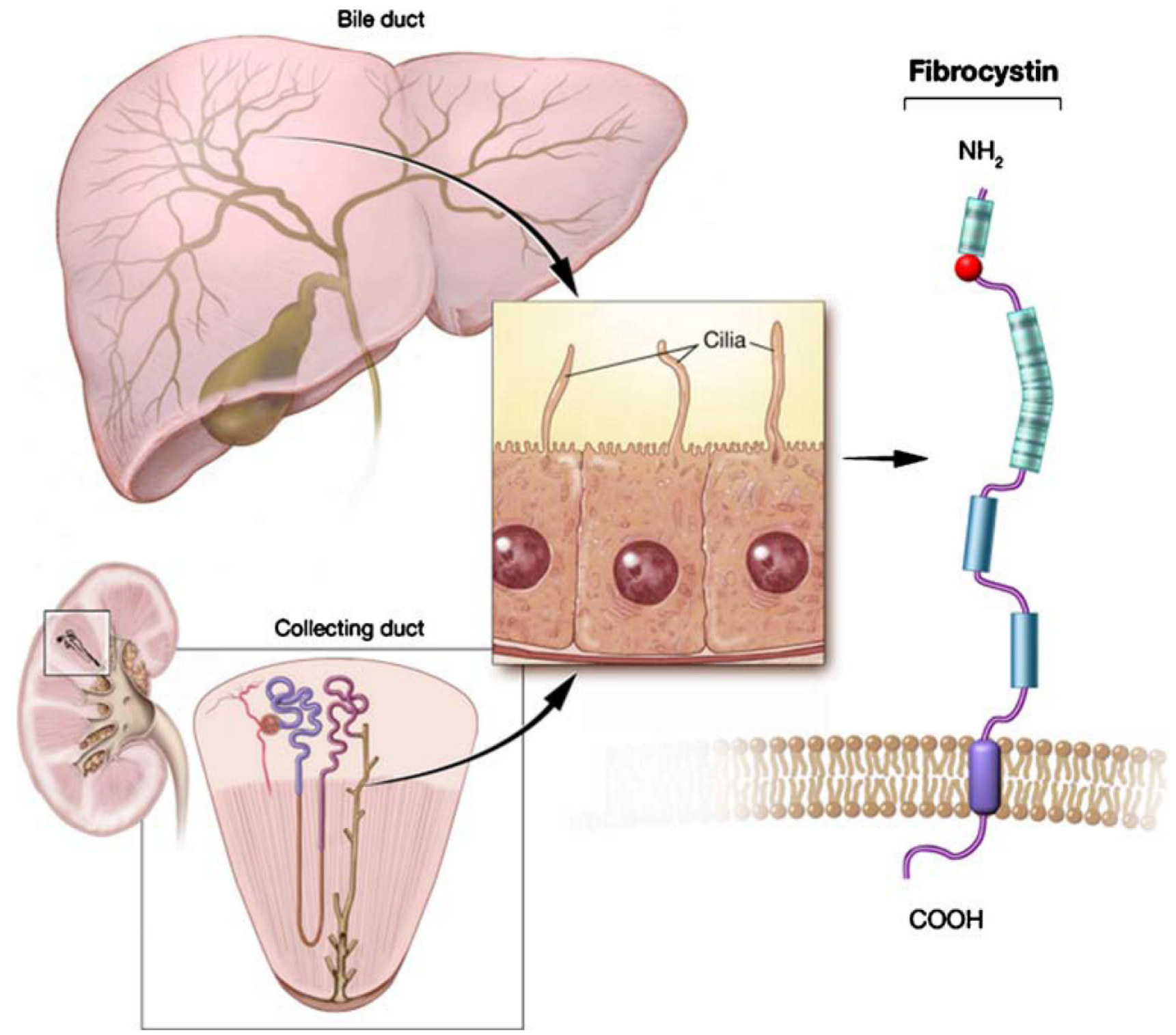

Fig. 2.

Diagram demonstrates the ultrastructure of the fibrocystin (polycystin) molecule, which is expressed in the cilia of both the bile ducts of the liver and collecting ducts of the kidney 


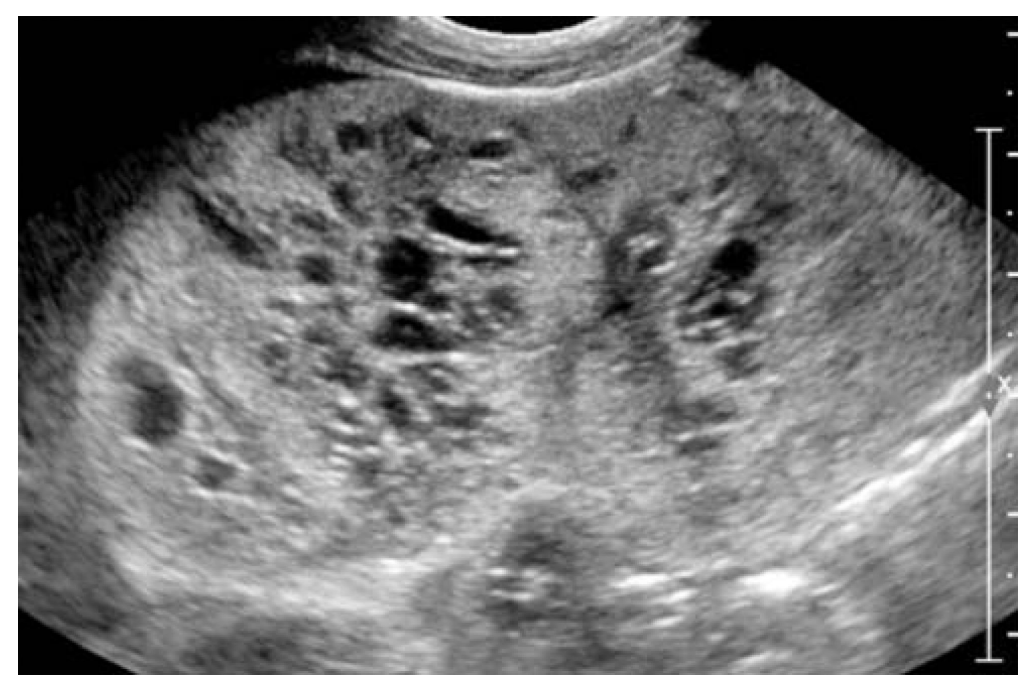

Fig. 3.

US image of a newborn with a perinatal ARPKD/CHF demonstrates an enlarged and diffusely echogenic right kidney secondary to numerous corticomedullary cysts (courtesy of Dr. Yair Anikster, Israel) 


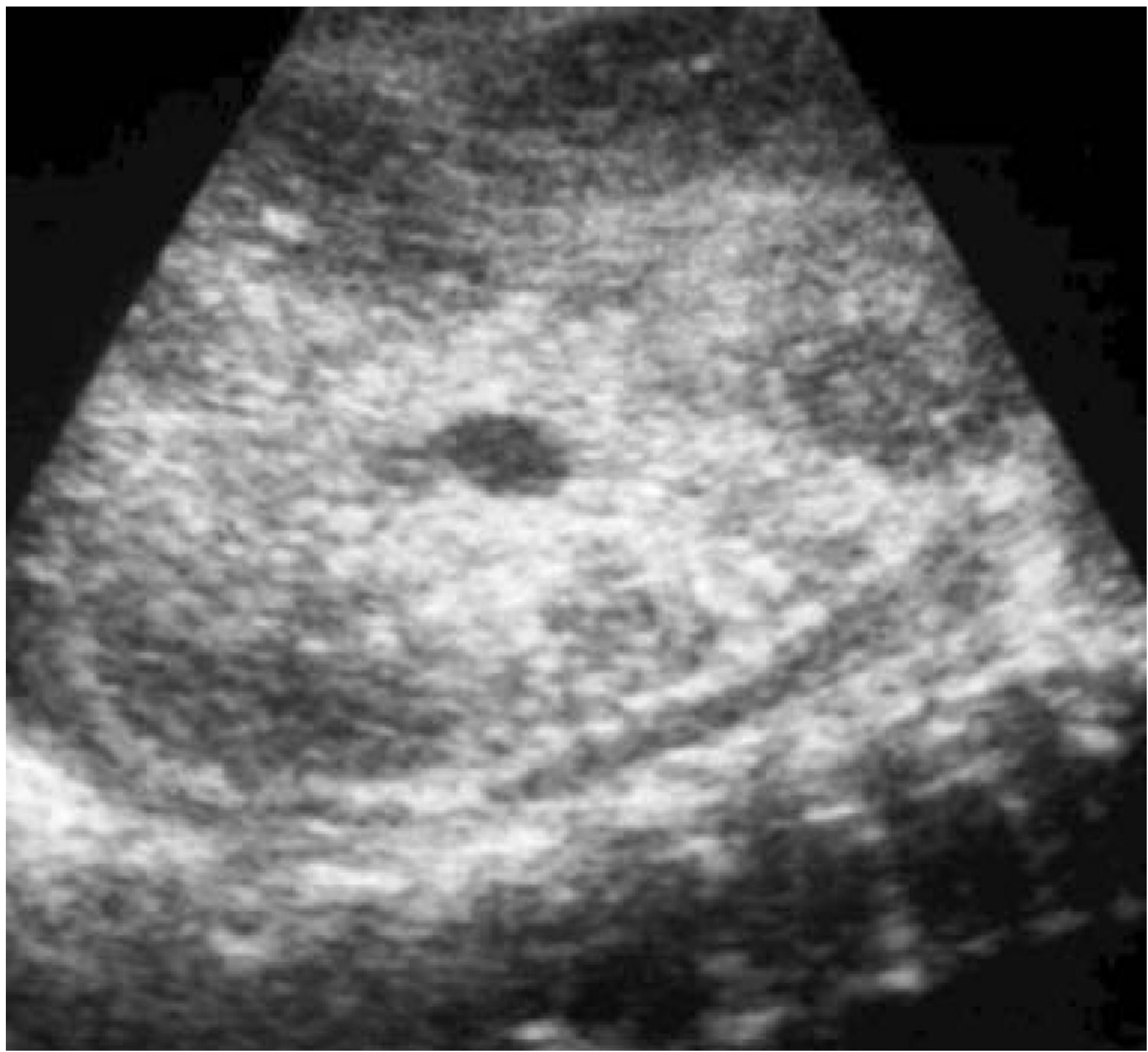

Fig. 4.

US image of a fetus with ARPKD/CHF demonstrates an enlarged echogenic right kidney (courtesy of Dr. Paula Woodward, University of Utah, Salt Lake City, UT) 

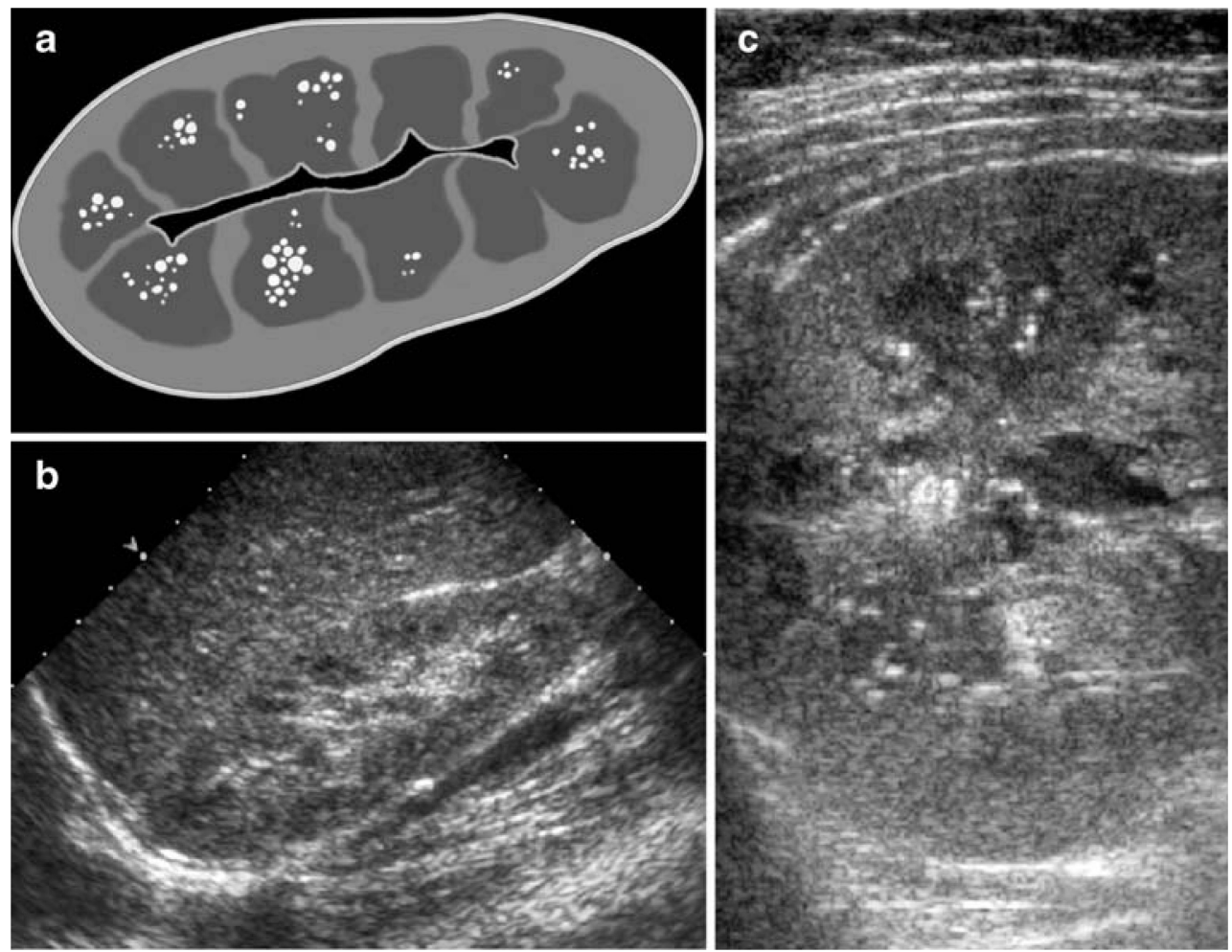

Fig. 5.

A 10-year-old girl with ARPKD/CHF. a Drawing represents partial medullary involvement in ARPKD. b Sagittal US image obtained with a conventional 4-MHz probe shows a normal-size right kidney with a preserved cortex and a mildly echogenic renal medulla. c High-resolution US image obtained with a 6-MHz probe demonstrates a few microcysts and echogenic foci within the medulla and a preserved renal cortex 

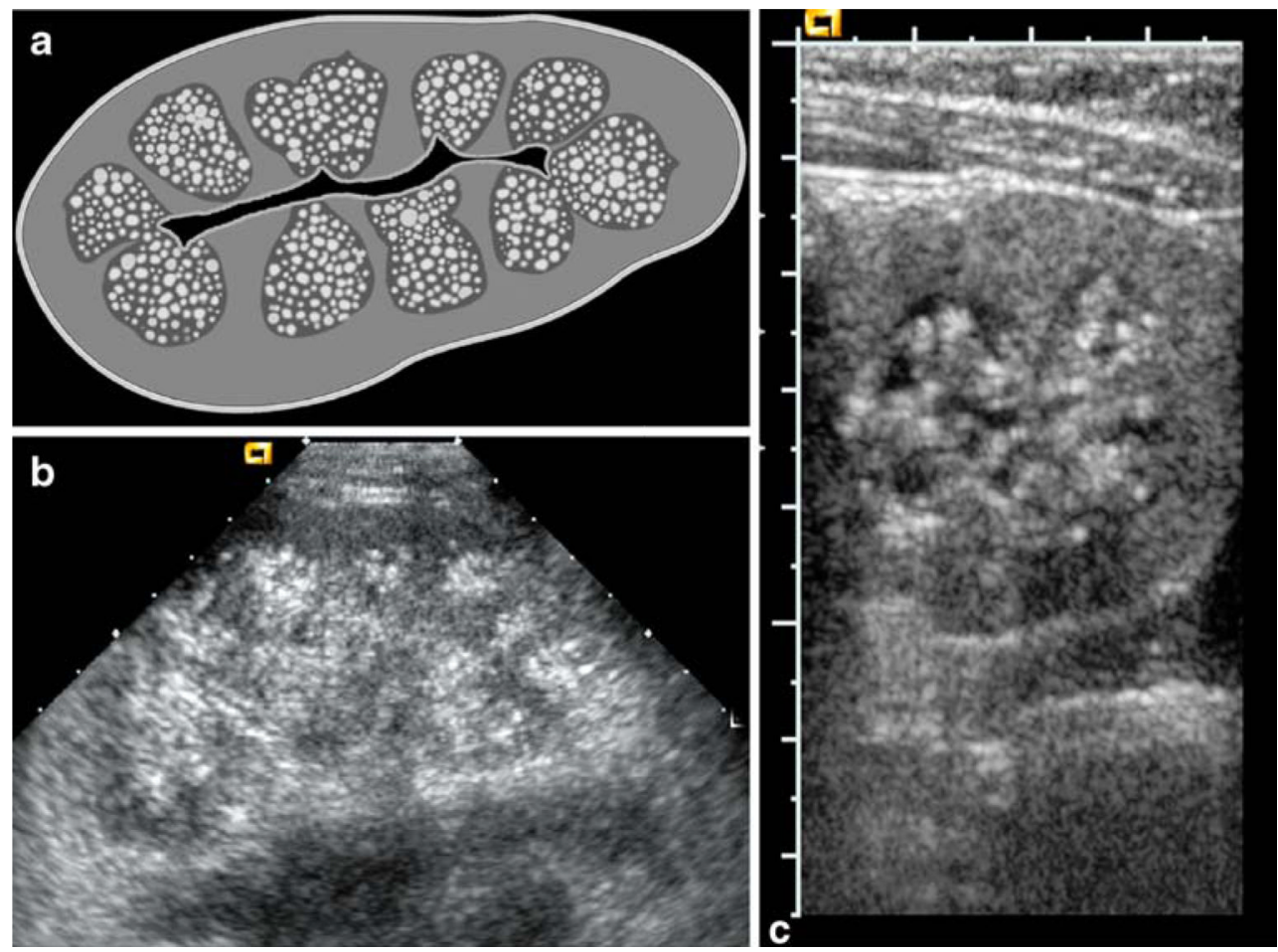

Fig. 6.

A 10-year-old boy with ARPKD/CHF. a Drawing represents complete medullary involvement in ARPKD. b Sagittal US image obtained with a conventional 4-MHz probe shows a mildly enlarged right kidney with a preserved cortex but significantly echogenic renal medulla. $\mathbf{c}$ High-resolution US image obtained with a 6-MHz probe demonstrates numerous microcysts and echogenic foci in the medulla with a preserved cortex 

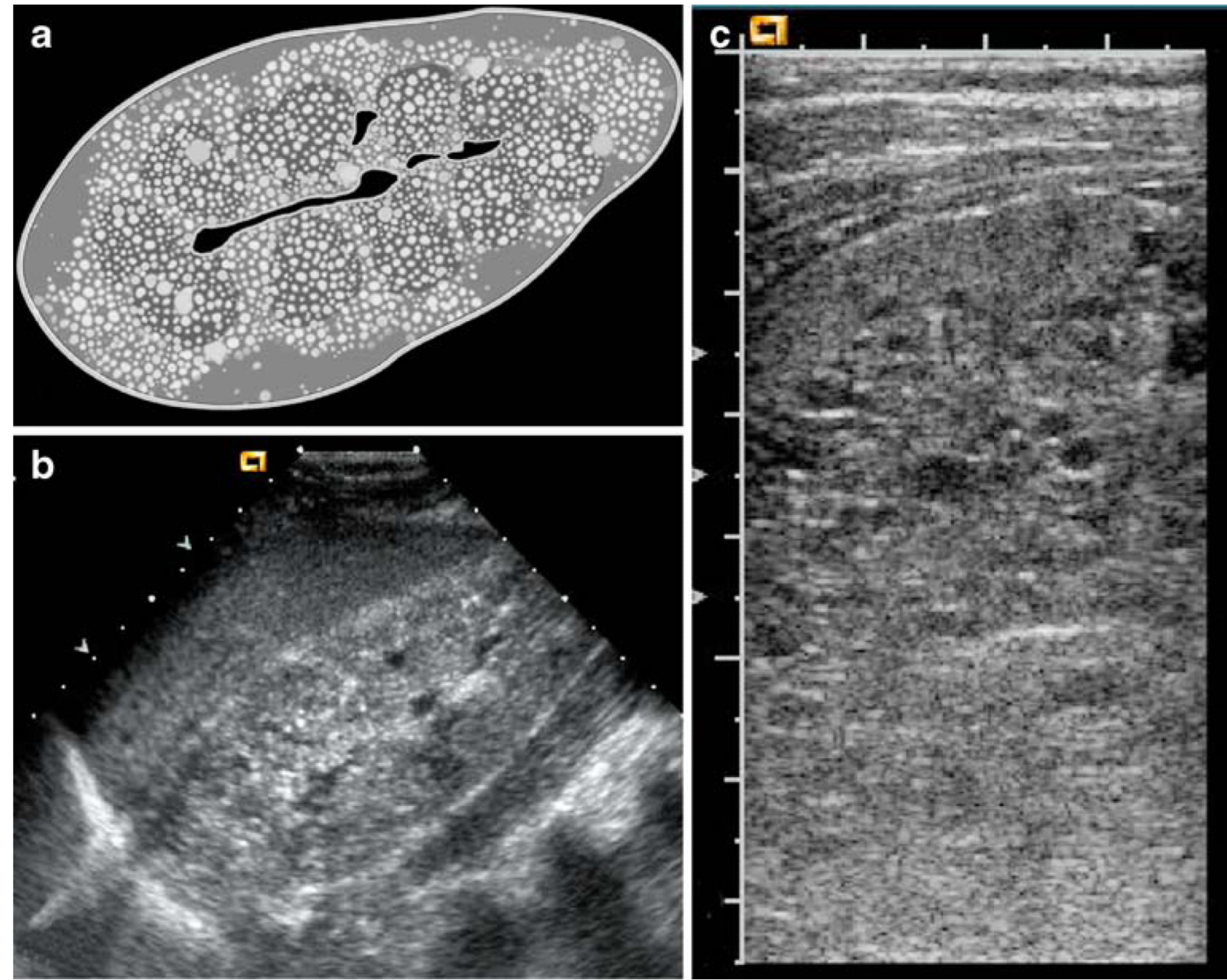

Fig. 7.

An 8-year-old boy with ARPKD/CHF. a Drawing represents complete medullary involvement with $<50 \%$ partial cortical involvement in ARPKD. b Sagittal US image obtained with a conventional 4-MHz probe shows a diffuse moderately echogenic right kidney with mild corticomedullary distinction loss. $\mathbf{c}$ High-resolution US image obtained with a 6-MHz probe demonstrates numerous microcysts invading the entire medulla and partially invading the cortex $(<50 \%)$ with a few echogenic foci 

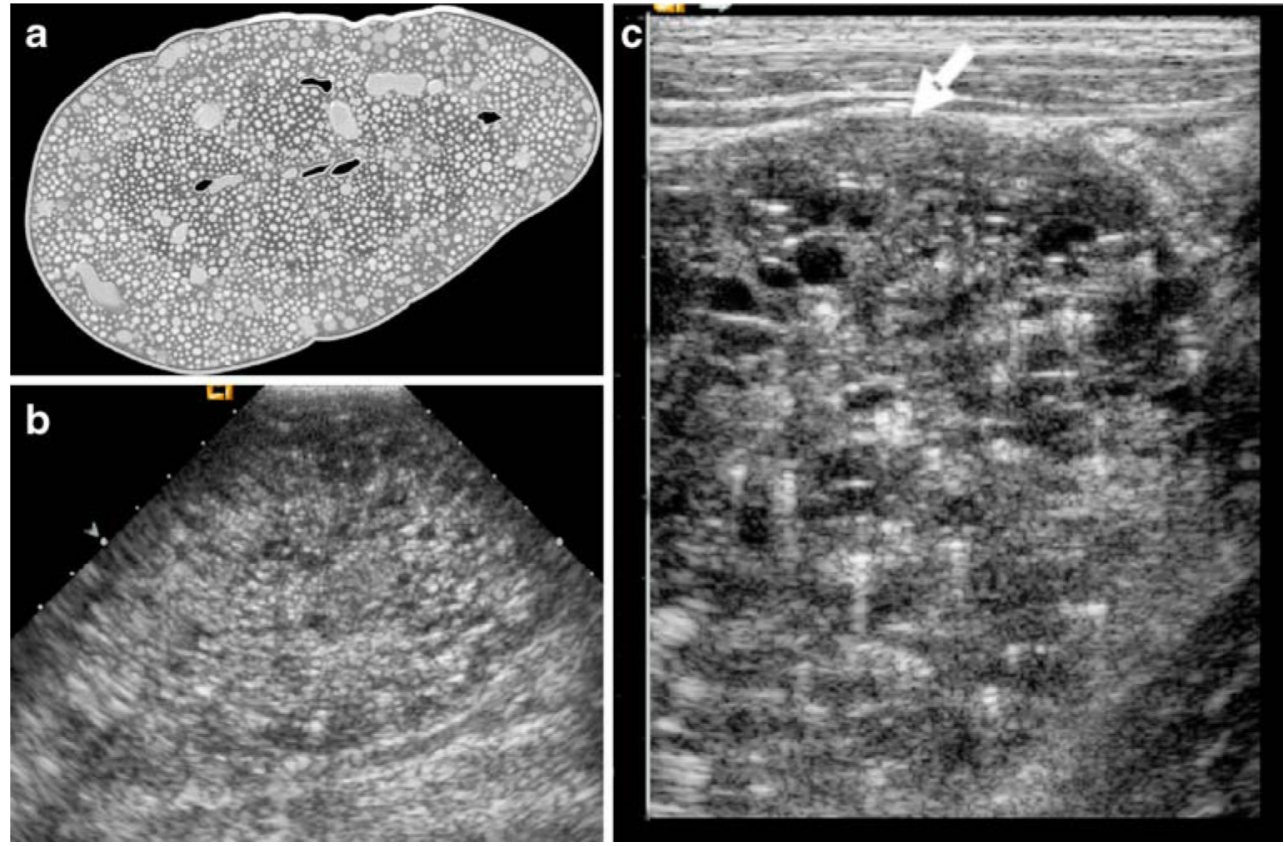

Fig. 8.

An 8-year-old girl with ARPKD/CHF. a Drawing represents complete medullary involvement with $>50 \%$ cortical involvement in ARPKD. b Sagittal US image obtained with a conventional 4-MHz probe shows a diffuse severely echogenic right kidney with complete corticomedullary distinction loss. $\mathbf{c}$ High-resolution US image obtained with a 6-MHz probe demonstrates numerous microcysts with tiny echogenic foci invading the medulla and cortex nearly totally $(>50 \%)$ with a thin preserved cortex (arrow) 


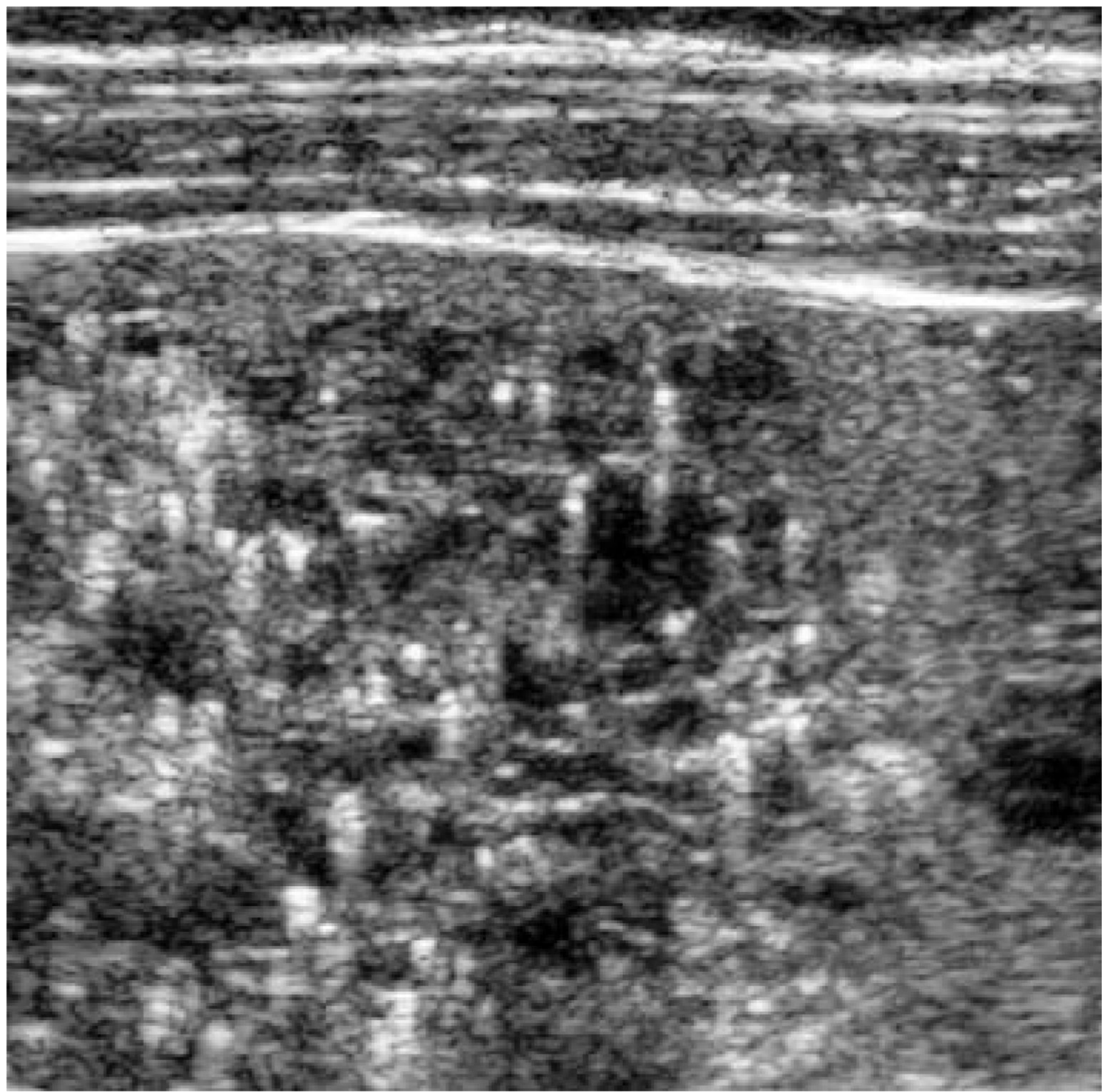

Fig. 9.

An 8-year-old boy with ARPKD/CHF. US image with a 7-MHz probe shows clusters of medullary echogenic foci without acoustic shadowing 

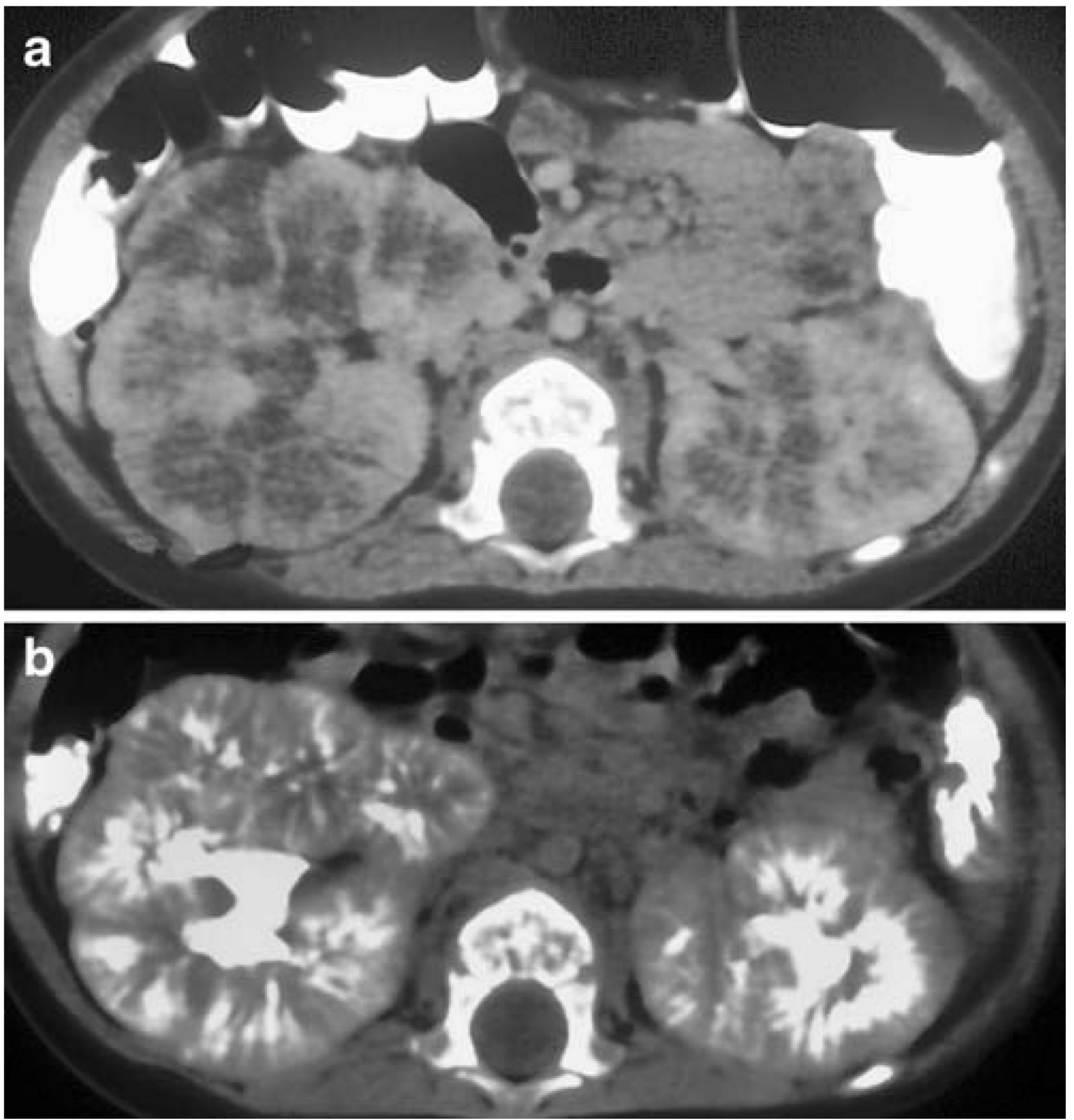

Fig. 10.

A 7-year-old girl with ARPKD/CHF. a Axial contrast-enhanced CT image in nephrographic phase demonstrates peripheral enhancement of the kidneys. b The 7-min delayed-phase image shows striated appearance of the kidneys as the excreted contrast material fills the dilated collecting ducts 

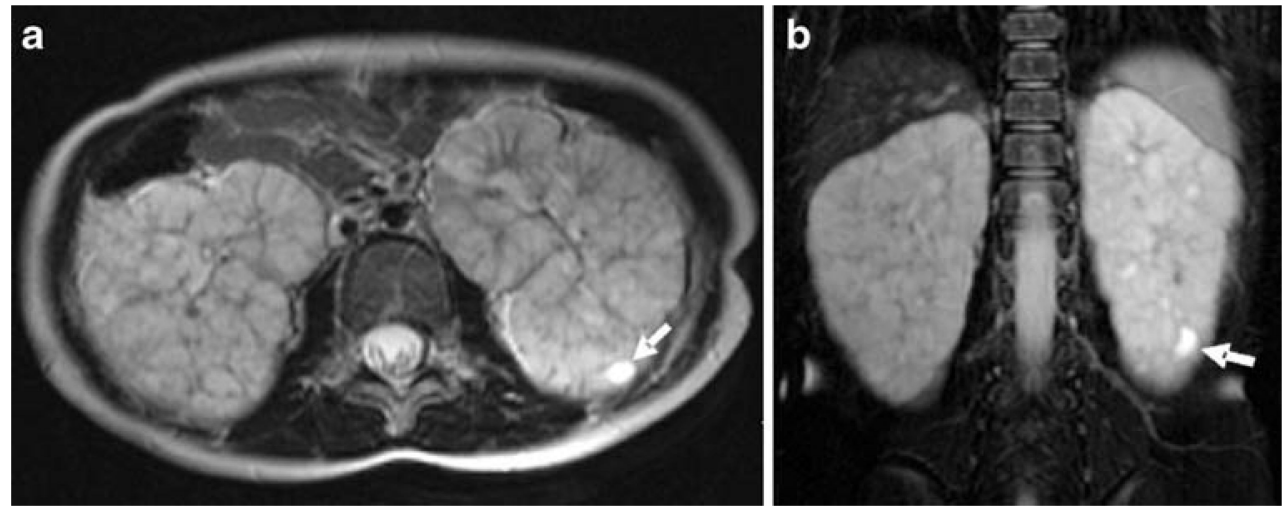

Fig. 11.

A 9-year-old girl with ARPKD/CHF. Axial (a) and coronal (b) T2-W MR images show bilateral diffusely enlarged kidneys with high signal intensity secondary to numerous corticomedullary microcysts. A macrocyst is seen at the lower pole of the left kidney (arrows) 

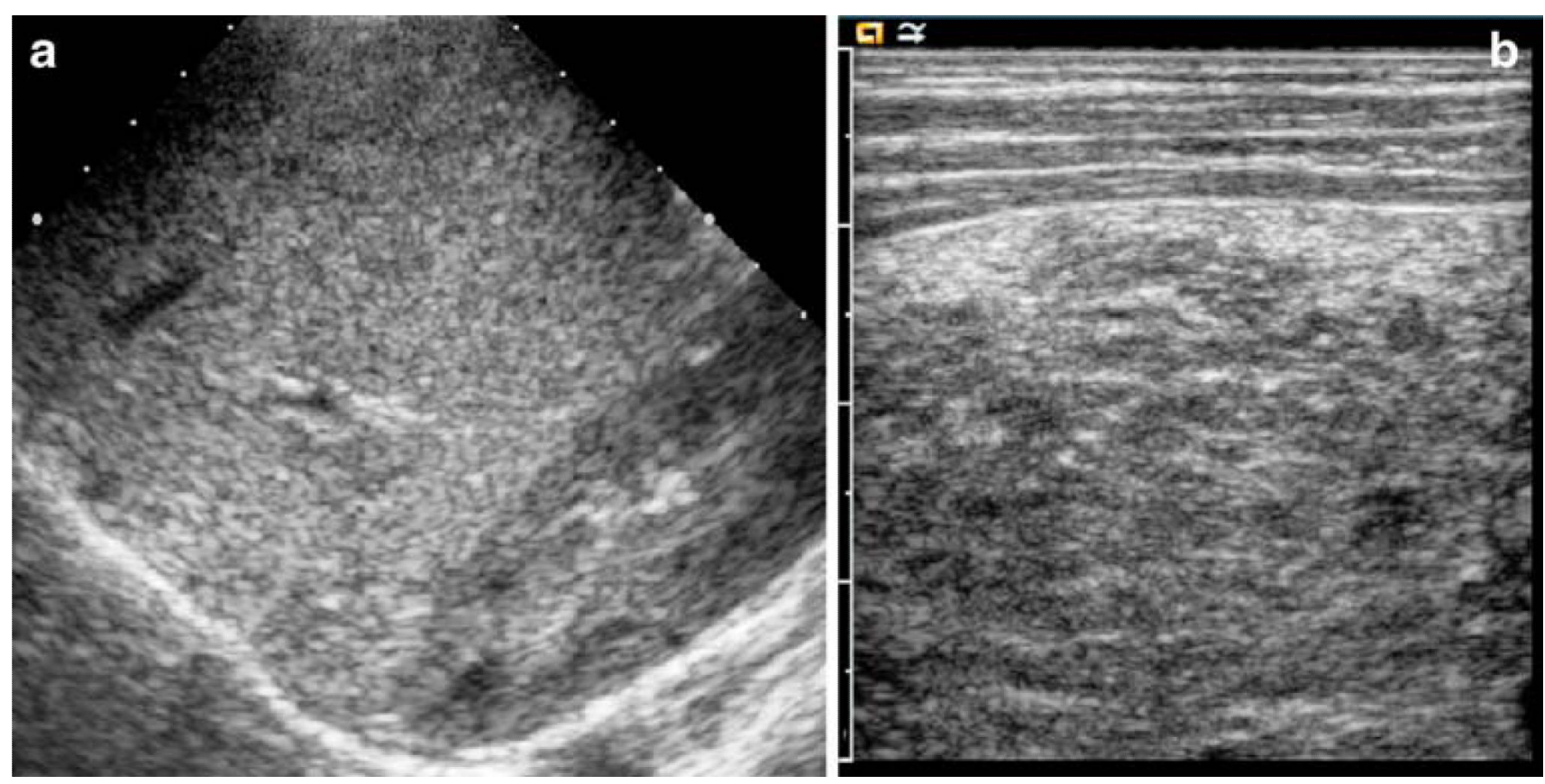

Fig. 12.

A 10-year-old boy with CHF. a Sagittal US image obtained with a conventional 4-MHz probe shows a severely echogenic liver compared with the right kidney secondary to fibrosis. b High-resolution US image obtained with a 6-MHz probe demonstrates significantly coarsened liver parenchymal echotexture 


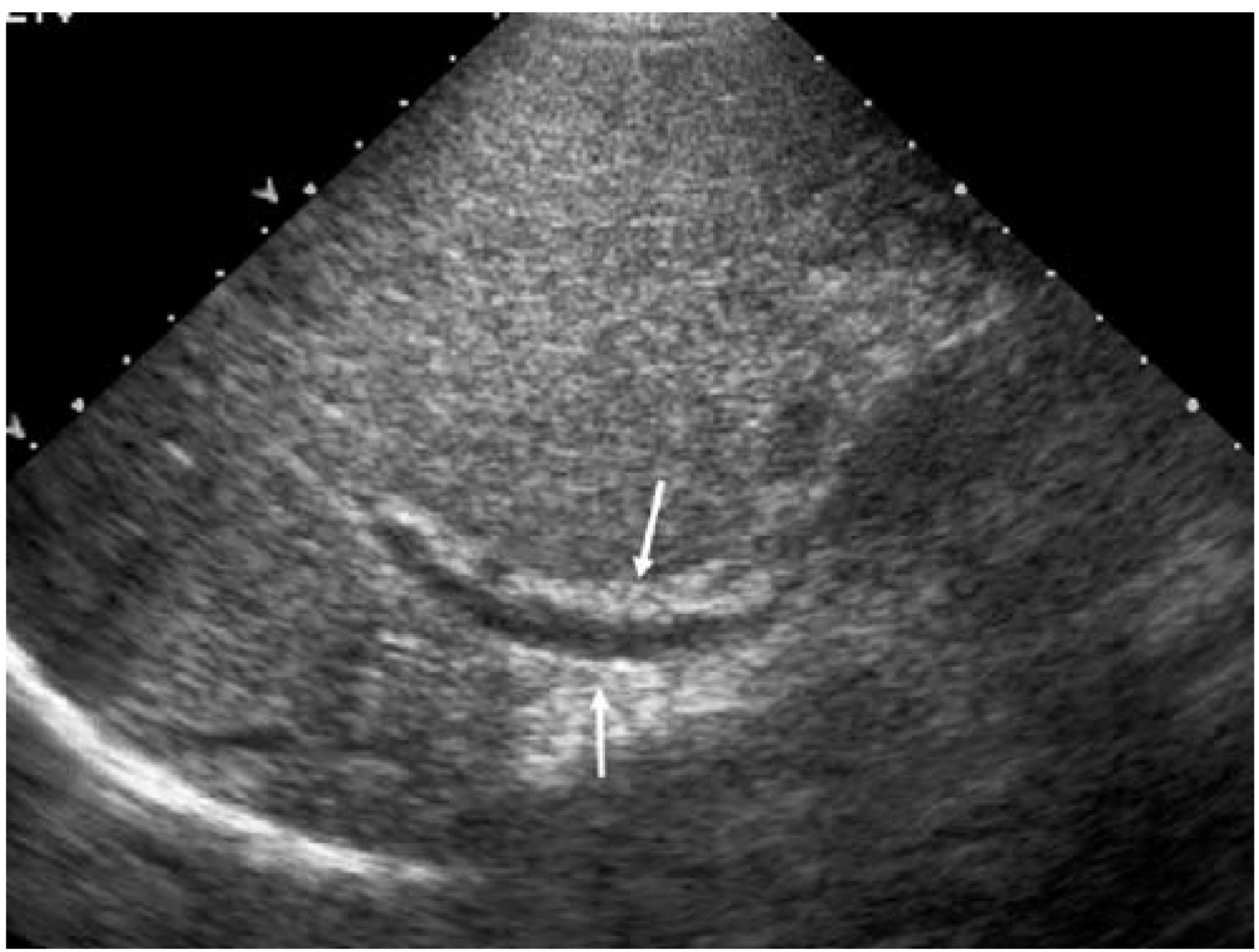

Fig. 13.

A 16-year-old-boy with CHF. US image obtained with a conventional 4-MHz probe shows moderately echogenic liver parenchyma and thickened periportal space (arrows) consistent with fibrosis 

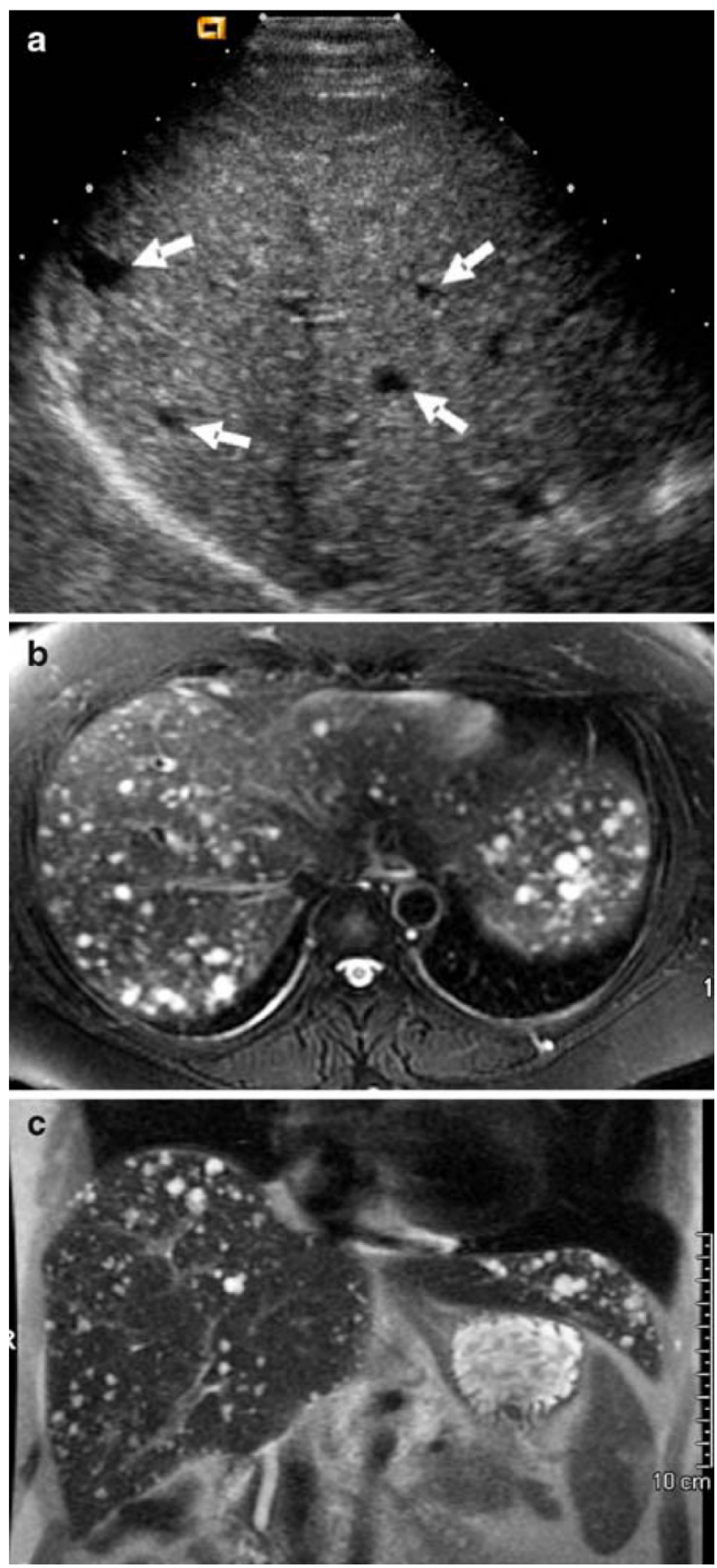

Fig. 14.

A 39-year-old woman with CHF. a US image obtained with a conventional 4-MHz probe shows numerous hypoechogenic lesions consistent with hepatic cysts (arrows). b, c T2-W MR images (b axial, c coronal) show an enlarged liver containing numerous cystic lesions along the course of the intrahepatic biliary ducts 

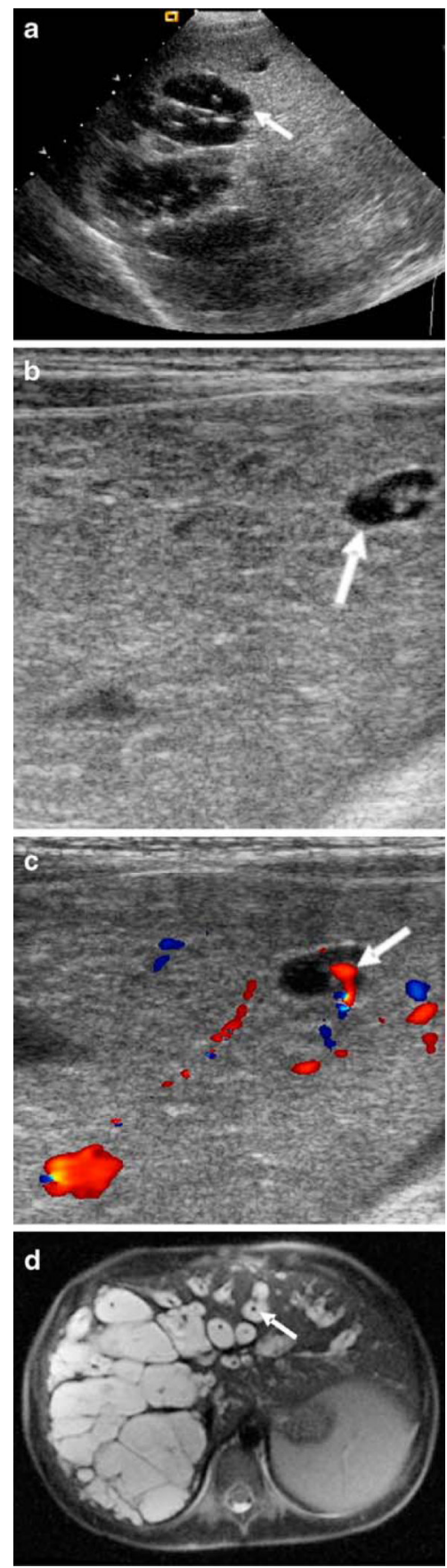

Fig. 15.

A 7-year-old boy with CHF and Caroli disease (Caroli syndrome). a US image obtained with a conventional 4-MHz probe shows clusters of macrocysts within the liver consistent with dilated intrahepatic bile ducts (arrow) and increased liver parenchymal echogenicity. b High-resolution US image obtained with a 6-MHz probe demonstrates a dilated intrahepatic bile duct with a central dot (arrow) and coarsened liver parenchyma consistent with fibrosis. c Color Doppler US image shows the flow within the central dot representing portal venous flow (arrow). d Axial T2-W MR image shows numerous biliary cysts within the liver parenchyma, some of which demonstrate the central portal vein (arrow) 

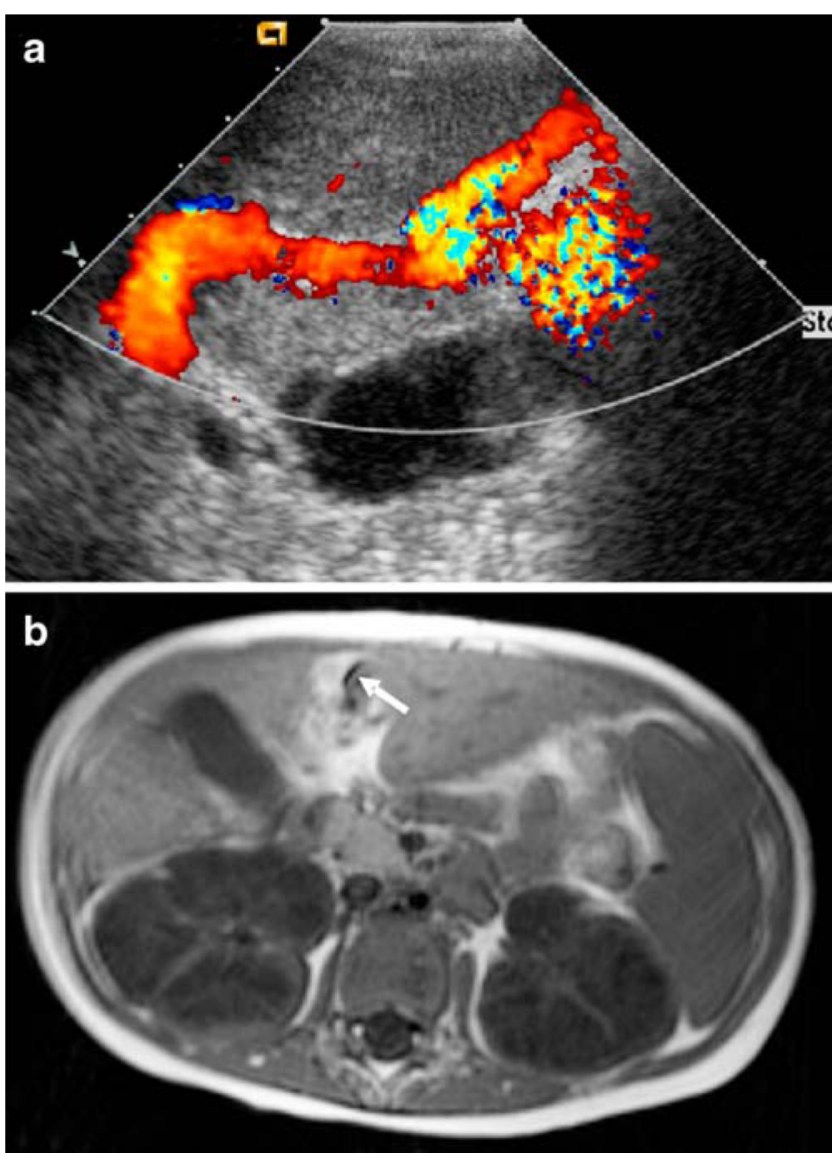

Fig. 16.

A 51/2-year-old boy with CHF. a Color Doppler US image shows flow in the patent umbilical vein secondary to PH. b Axial T1-W unenhanced MR image demonstrates the patent umbilical vein (arrow) 


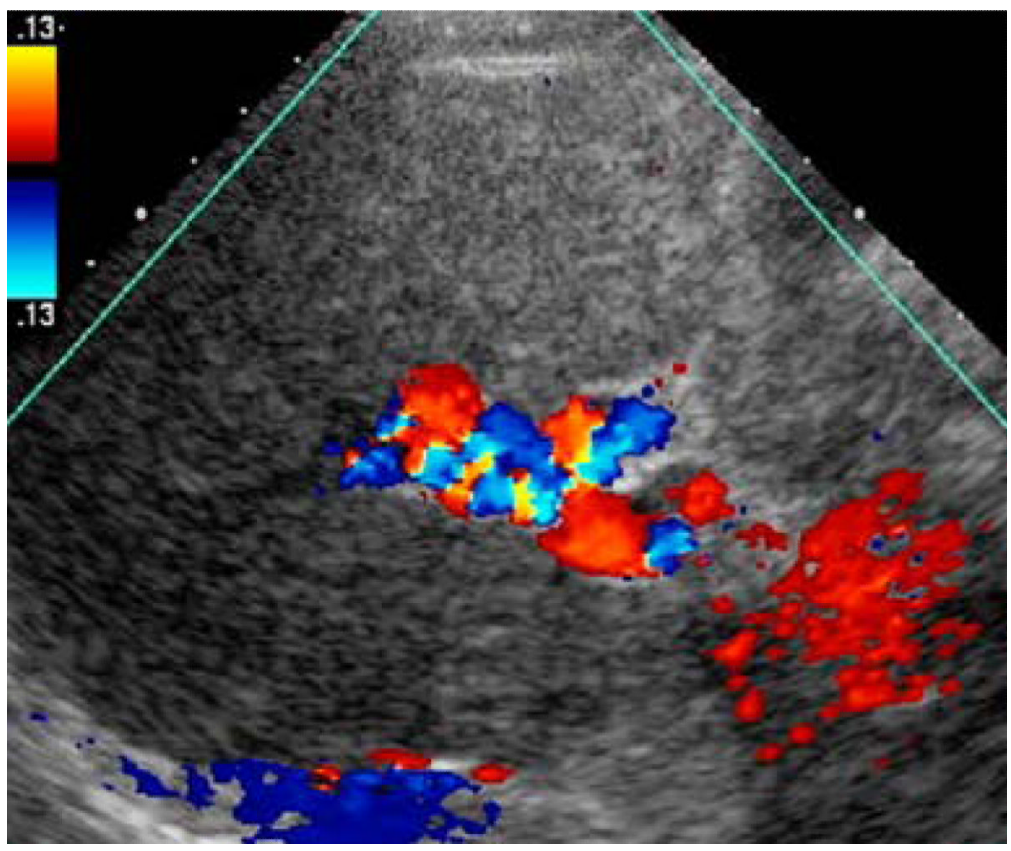

Fig. 17.

A 35-year-old man with ARPKD/CHF. Color Doppler US image shows multiple vessels at the porta hepatis with a patent portal vein resembling the cavernomatous transformed vessels occurring secondary to portal vein obstruction 

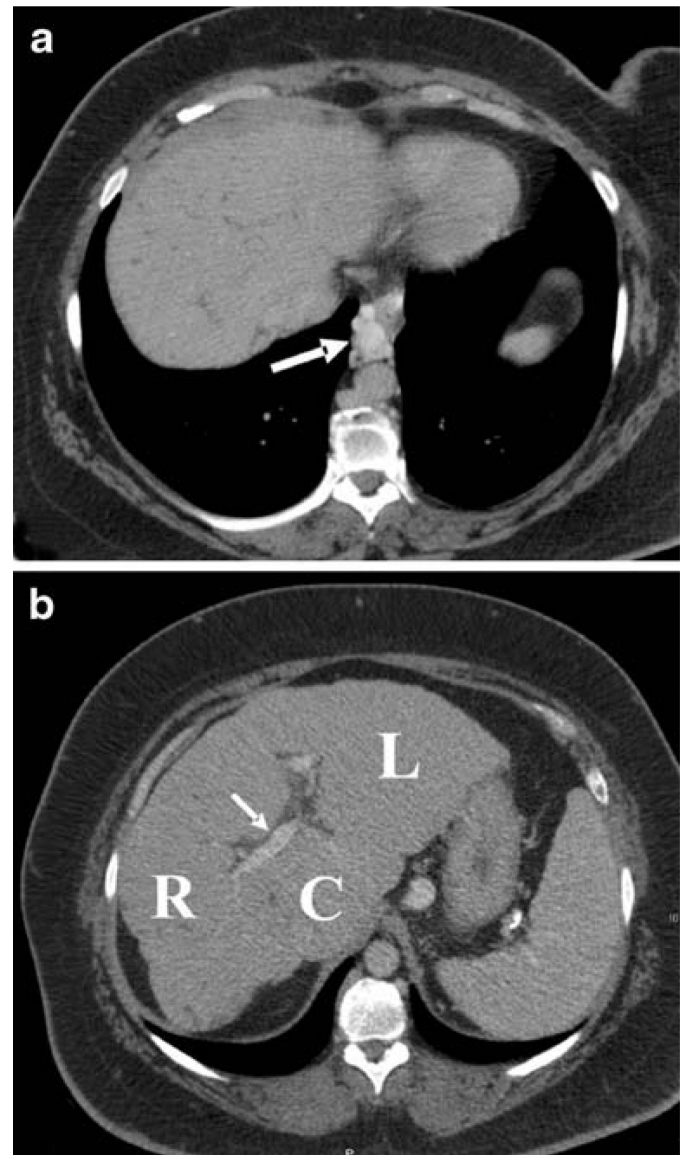

Fig. 18.

A 50-year-old woman with CHF. Axial contrast-enhanced CT images show (a) paraesophageal varicose veins (arrow) and (b) liver contour irregularity, atrophy of the right lobe $(R)$, hypertrophy of the left $(L)$ and caudate $(C)$ lobes, mild periportal thickening (arrow), and splenomegaly, all of which are secondary to $\mathrm{PH}$ 

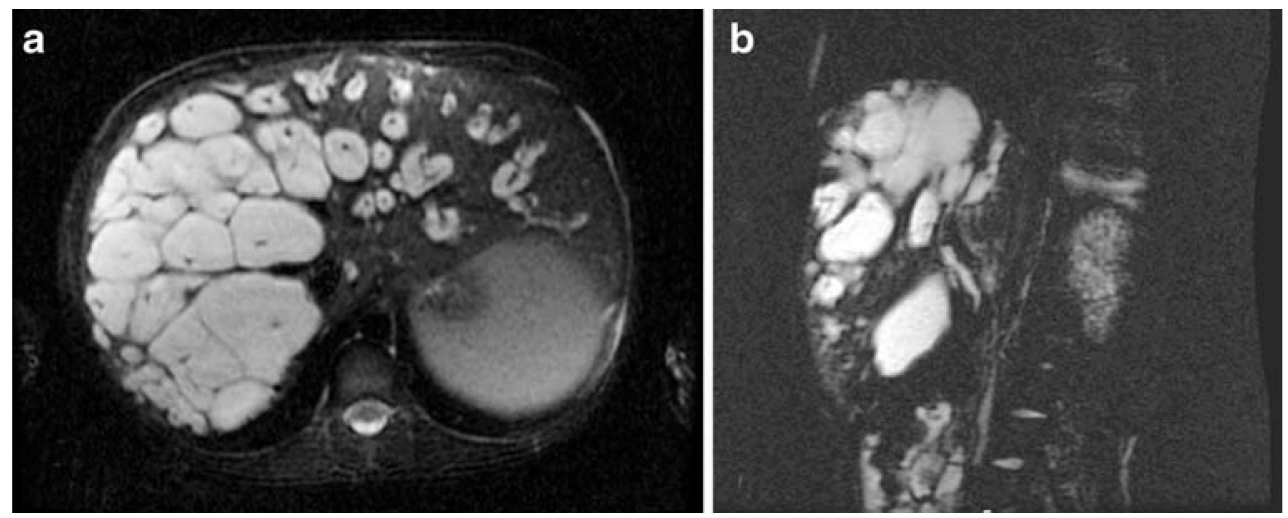

Fig. 19.

A 7-year-old boy with Caroli syndrome (same patient as in Fig. 15). Axial (a) and reconstructed oblique (b) MR cholangiography images demonstrate dilated intrahepatic biliary ducts with the central dot sign 


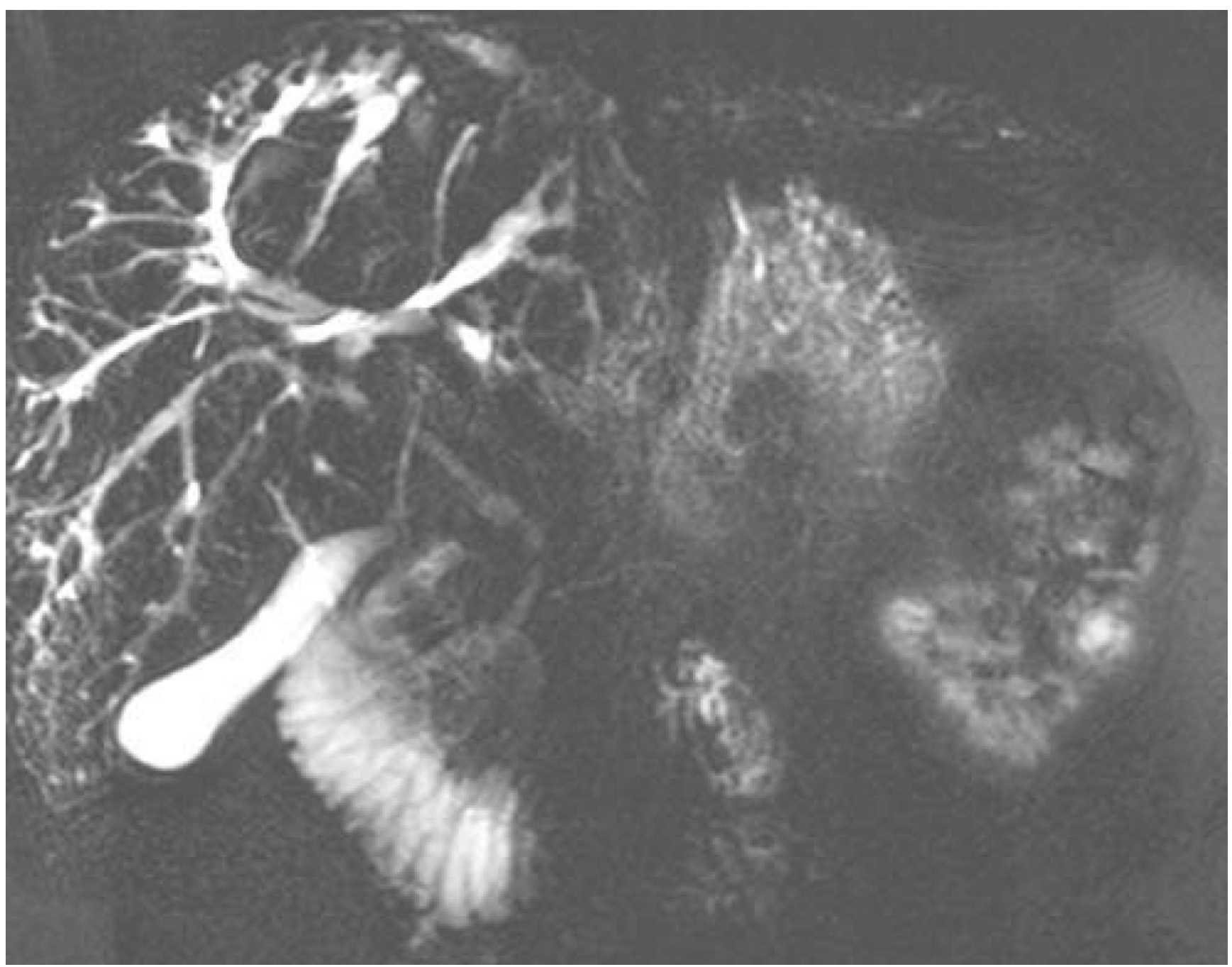

Fig. 20.

A 25-year-old man with ARPKD/CHF. Coronal MR cholangiography image demonstrates dilated peripheral intrahepatic biliary ducts and adjacent small cysts 